\title{
Evaluación gastrointestinal en los pacientes con fiebre mediterránea familiar con dolor abdominal crónico y tratados con colchicina: serie de casos
}

\author{
Gastrointestinal evaluation in colchicine-treated familial Mediterranean fever \\ patients with chronic abdominal pain: Cases series
}

\author{
Dra. Sezin Akman ${ }^{a}$, Dr. Selcuk Yazicib y Dr. Can Ozturk
}

\section{RESUMEN}

El objetivo de este estudio fue evaluar a los pacientes con fiebre mediterránea familiar (familial Mediterranean fever, FMF) y dolor abdominal crónico resistentes al tratamiento con colchicina. Se incluyó a 48 pacientes diagnosticados en nuestro consultorio de reumatología pediátrica que tenían dolor abdominal a pesar del tratamiento con colchicina. A todos los pacientes se los derivó a un gastroenterólogo pediátrico. Se registraron las características del dolor, tales como aparición, duración y frecuencia; se planificó una endoscopía digestiva para obtener un diagnóstico diferencial.

Se determinó la presencia de una mutación del gen $M E F V$ en 46 pacientes. La mediana de la duración del tratamiento fue de 2,8 años. Aproximadamente el $60 \%$ de los pacientes tenían dolor abdominal todos los días o de dos a tres veces a la semana; en el 73\% de los casos, duró menos de tres horas. A 41 pacientes se les realizó una endoscopía digestiva alta.

La gastroduodenitis es un hallazgo frecuente en los pacientes con FMF y dolor abdominal persistente a pesar del tratamiento. Los pacientes con los puntajes más altos de severidad de la enfermedad tenían inflamación digestiva grave.

Palabras clave: dolor abdominal, gastroenterología, diagnóstico, fiebre mediterránea familiar.

http: / / dx.doi.org/10.5546/ aap.2018.e649

Texto completo en inglés:

http: / / dx.doi.org/10.5546/ aap.2018.eng.e649

Cómo citar: Akman S, Yazici S, Ozturk C. Evaluación gastrointestinal en los pacientes con fiebre mediterránea familiar con dolor abdominal crónico y tratados con colchicina: serie de casos. Arch Argent Pediatr 2018;116(5):e649-e654.

a. Facultad de Medicina de Balıkesir Üniversitesi, Departamento de Pediatría, Unidad de Gastroenterología Pediátrica, 10145 Balıkesir, Turquía.

b. Facultad de Medicina de Balıkesir Üniversitesi, Departamento de Pediatría, 10145 Balıkesir, Turquía.

c. Ministerio de Salud de Esmirna, Hospital Universitario y de Investigación de Tepecik, Unidad de Inmunología y Reumatología Pediátricas, 35110 Esmirna, Turquía.

Correspondencia: Dra. Sezin Akman: aa.sezin@gmail.com

Financiamiento: Ninguno.

Conflicto de intereses: Ninguno que declarar.

Recibido: 1-10-2017

Aceptado: 4-3-2018

\section{INTRODUCCIÓN}

La fiebre mediterránea familiar (familial Mediterranean fever, FMF por sus siglas en inglés) es la enfermedad autoinflamatoria más frecuente en las personas descendientes de pueblos del Mediterráneo oriental. El gen causante de la FMF (MEFV) se encuentra en el cromosoma 16; el estado homocigoto en la mutación M694V está asociado con una forma más grave de la enfermedad. Los síntomas característicos son la fiebre acompañada por dolor abdominal, torácico o articular y compromiso del escroto. La mayoría de los síntomas se resuelven de manera espontánea dentro de las 12-24 horas. La complicación más devastadora es la amiloidosis, que, en última instancia, causa insuficiencia renal terminal. ${ }^{1-3} \mathrm{El}$ peritoneo es la membrana serosa más comúnmente afectada. Por lo tanto, el dolor abdominal es el síntoma más frecuente en los pacientes. La mayor parte del tiempo, a los pacientes con FMF se los deriva a un gastroenterólogo antes del diagnóstico. El dolor abdominal podría ser secundario a enfermedades asociadas con la FMF, tales como enfermedad intestinal inflamatoria o amiloidosis. ${ }^{1,4,5} \mathrm{La}$ colchicina es muy eficaz como tratamiento ya que previene el desarrollo de ataques y amiloidosis. En general, los síntomas desaparecen con el tratamiento debido al control de la inflamación. Sin embargo, en algunos casos, los síntomas podrían ser resistentes al tratamiento aunque se logre el control adecuado de los marcadores inflamatorios. Pueden presentarse dificultades en los pacientes que tienen enfermedades digestivas asociadas o que se superponen. ${ }^{1,3,4,6}$

Nuestro objetivo fue evaluar a los pacientes con dolor abdominal crónico resistentes al tratamiento con colchicina.

\section{MÉTODOS}

Este estudio se realizó en 48 pacientes con FMF entre enero de 2011 y junio de 2012. El diagnóstico se determinó conforme a los criterios de Livneh. ${ }^{2}$ 
A todos los pacientes se los controló en la Unidad de Reumatología Pediátrica y se los trató con colchicina durante, al menos, seis meses. La dosis inicial de colchicina fue de 0,5-1 mg/ día. Se aumentó la pauta posológica gradualmente hasta llegar a los $2 \mathrm{mg} /$ día en los pacientes resistentes. En el grupo del estudio se incluyó a los pacientes con dolor abdominal crónico atendidos en la Unidad de Gastroenterología Pediátrica. Se calculó el puntaje de severidad de la enfermedad (disease severity score, DSS) de todos los pacientes según los criterios del estudio de $\operatorname{Pras}^{7}$ y Roma III $^{8}$ para la evaluación del dolor abdominal.

El estudio fue aprobado por el Comité de Ética (14 de enero, 2011) y se obtuvo el consentimiento informado de todos los padres. Se excluyó del estudio a todos los niños con otra enfermedad indicativa de abdomen agudo. Se excluyó a los pacientes con infecciones, inmunodeficiencias y alergias alimentarias detectadas por coprocultivo, análisis inmunológico, prueba intraepidérmica y dieta de eliminación (Figura 1). Se evaluaron las características de los pacientes, las mutaciones del gen $M E F V$, la aparición, frecuencia y duración del dolor, los resultados de los análisis de laboratorio para infección por Helicobacter pylori (H. pylori) y celiaquía.

Se obtuvo el ADN de sangre periférica mediante protocolos estándar (equipo Invisorb ${ }^{\circledR}$ Spin Blood, STRATEC, Alemania). Se realizaron análisis moleculares dentro del marco de los análisis genéticos habituales. Se estudió la

FIGURA 1. Criterios de inclusión y exclusión de los pacientes

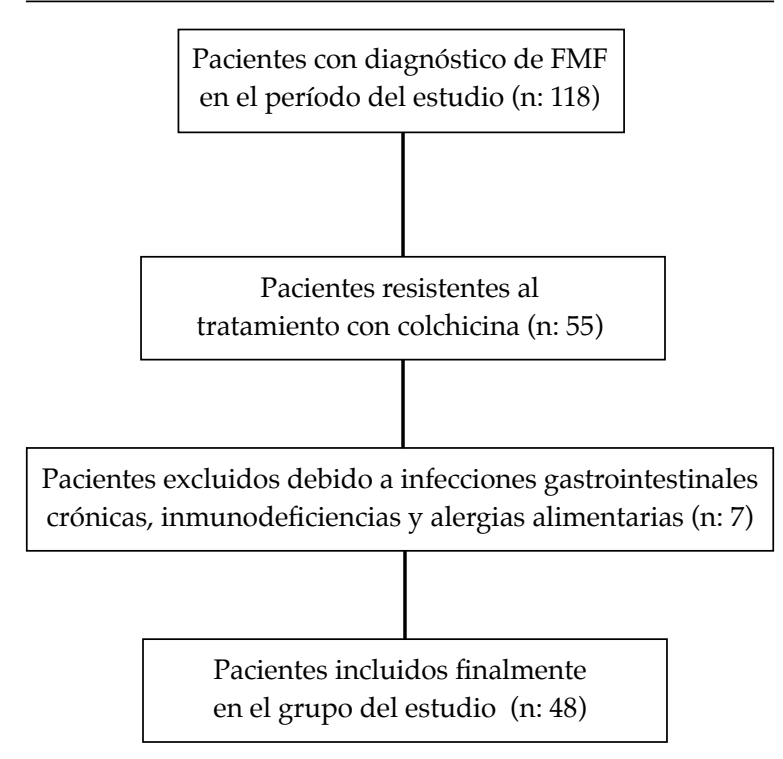

mutación del gen $M E F V$ con el método de PCR por hibridación inversa. Se diagnosticó infección por H. pylori mediante endoscopía, prueba del aliento con urea marcada y detección del antígeno en heces. Se planificó la endoscopía para los pacientes con FMF resistentes a la colchicina. Cuarenta y una de las familias de los pacientes aceptaron que se realice este procedimiento. Se realizaron endoscopías digestivas altas y bajas con sedación, si estaba indicado, después de obtener el consentimiento informado. Se obtuvieron al menos dos piezas de biopsia del duodeno al esófago y se evaluaron las características histológicas.

Para eliminar el H. pylori, se administró un tratamiento triple con un inhibidor de la bomba de protones (IBP) más amoxicilina y claritromicina o metronidazol durante 7-14 días. ${ }^{9}$ Se administró el IBP a los pacientes con gastritis / esofagitis; y se agregó el metronidazol, además de antiácidos, para el tratamiento de la duodenitis. Se llamó a los pacientes de manera periódica para evaluar la respuesta al tratamiento durante seis meses.

Se usó el programa SPSS (Chicago, EE. UU., versión 15.0) para identificar los datos demográficos y clínicos. Los valores del estudio se describen como media \pm desviación estándar, porcentaje (\%) y número (n). Se utilizaron la mediana y los intervalos cuando no se disponía de datos con distribución normal.

\section{RESULTADOS}

En la Figura 1, se presenta el diagrama de flujo de los pacientes. La media de edad fue de 11,7 $\pm 3,2$ años; $20 / 48$ (41,7\%) eran varones. La mediana de la duración del tratamiento con colchicina fue de 2,8 años, y el dolor apareció, en promedio, $3,3 \pm 1,8$ meses después del inicio de la colchicina. El dolor persistió en el $75 \%$ de los pacientes durante, al menos, tres meses; en el $90 \%$, la frecuencia del dolor fue diaria o, al menos, tres veces a la semana; en aproximadamente el $75 \%$, duró menos de tres horas; y en el $80 \%$, la ubicación principal fue el abdomen completo o el epigastrio. El síntoma más frecuente que acompañó el dolor fueron las náuseas $(56,2 \%)$. Los resultados de la prueba del aliento con urea marcada y la serología de la celiaquía fueron positivos en nueve pacientes $(18,7 \%)$ y en un paciente $(2,1 \%)$, respectivamente (Tabla 1$)$.

El 21\% de los pacientes tenían antecedentes familiares de FMF; y se detectó una mutación del gen $M E F V$ en 46 pacientes $(95,8 \%)$. El DSS 
promedio fue de $6,8 \pm 2,1$. Cabe destacar que todos los pacientes con el DSS más alto tenían inflamación digestiva (Tabla 2). Se realizó una endoscopía digestiva alta en 41 pacientes $(85,4 \%)$ y una colonoscopía en tres. El hallazgo histológico más prominente fue la gastroduodenitis (Figuras 2 y 3). Todos los resultados fueron normales en los pacientes sometidos a la colonoscopía. El 67\% de los pacientes respondió al tratamiento.

\section{DISCUSIÓN}

En este estudio, se evaluó a los pacientes con FMF que recibieron dosis efectivas de colchicina con dolor abdominal persistente sin la presencia de otros hallazgos relacionados con la FMF durante el seguimiento. A estos pacientes se los derivó al Departamento de Gastroenterología Pediátrica para la evaluación del dolor abdominal. Aproximadamente cuatro de cada cinco pacientes requirió una endoscopía. Las patologías digestivas fueron más frecuentes en los niños con DSS alto.

Según la definición de los pacientes, el dolor abdominal no estaba acompañado por dolor articular o fiebre, a veces ocurría todos los días y no se quejaron de dolor abdominal por FMF. Difería de los ataques de FMF "típicos", que podrían incluir peritonitis "real" o aumento de los parámetros de la fase aguda. En la mayoría de los pacientes, el dolor duró menos de lo esperado con la FMF. En estudios previos, se informó la dificultad para distinguir el dolor abdominal en el epigastrio o todo el abdomen de los ataques típicos. Dado que el dolor abdominal episódico afecta al 95\% de los pacientes con FMF, la mayoría consulta a gastroenterólogos. ${ }^{1,4,10}$

La cantidad de estudios que se centran en la investigación de la infección e inflamación digestivas en el diagnóstico diferencial del dolor abdominal en los pacientes con FMF, a pesar del tratamiento, es pequeña. Además de las publicaciones asociadas con enfermedad intestinal inflamatoria y mutación positiva del gen $M E F V$, se informó que algunos pacientes con colitis inflamatoria recibieron, en última instancia, el diagnóstico de FMF. ${ }^{6,11}$ En la bibliografía, no se cuenta con información suficiente sobre la evaluación de la mucosa digestiva en los niños con FMF. Se informó compromiso de la mucosa digestiva sin amiloidosis como manifestación relacionada con un ataque de FMF en el estudio

TABla 1. Características de los pacientes y del dolor abdominal

$\begin{array}{lc}\text { Sexo }(\mathrm{M} / \mathrm{F}, \mathrm{n}[\%]) & 20(41,7) / 28(58,3) \\ \text { Edad (media } \pm \mathrm{DE}) \text { (años) } & 11,7 \pm 3,2 \\ \text { Aparición del dolor (meses) } & 3,3 \pm 1,8 \\ \text { Duración del tratamiento con colchicina (años) } & 2,8(1-4,9) \\ \text { Prueba positiva del aliento con urea marcada (n [\%]) } & 9(18,7) \\ \text { Serología positiva para celiaquía (n [\%]) } & 1(2,1)\end{array}$

Características del dolor

\begin{tabular}{lclc} 
Frecuencia & $\mathbf{n}(\%)$ & Ubicación & $\mathbf{n}(\%)$ \\
\hline Todos los días & $13(27)$ & Epigastrio & $18(37,5)$ \\
Dos o tres veces a la semana & $17(35,4)$ & Abdomen completo & $19(39,6)$ \\
Una vez a la semana & $13(27)$ & Mesogastrio & $9(18,7)$ \\
Una o dos veces al mes & $5(10,4)$ & Retroesternón & $2(4,2)$ \\
\hline Duración & & Signos concurrentes \\
$<1$ hora & $24(50,0)$ & Náuseas & $27(56,2)$ \\
1-3 horas & $11(22,9)$ & Vómitos & $3(6,2)$ \\
3-12 horas & $5(10,4)$ & Náuseas + vómitos & $6(12,5)$ \\
$>12$ horas & $8(16,6)$ & Diarrea & $5(10,4)$ \\
& & Ninguno & $7(14,5)$ \\
\hline
\end{tabular}

*mediana, intervalos 
TABLA 2. Mutaciones del gen MEFV en relación con los hallazgos endoscópicos y puntajes de severidad de la enfermedad

\begin{tabular}{|c|c|c|c|c|c|c|c|c|}
\hline \multirow[t]{2}{*}{ Paciente } & \multirow[t]{2}{*}{$\begin{array}{l}\text { Mutación del gen } \\
M E F V \text { en el exón } 2 \\
\end{array}$} & \multirow[t]{2}{*}{$\begin{array}{c}\text { Mutación del gen } \\
M E F V \text { en el exón } 3\end{array}$} & \multirow[t]{2}{*}{$\begin{array}{c}\text { Mutación del gen } \\
M E F V \text { en el exón } 10\end{array}$} & \multicolumn{4}{|c|}{$\begin{array}{c}\text { Hallazgos } \\
\text { endoscópicos }\end{array}$} & \multirow[t]{2}{*}{ DSS $^{* *}$} \\
\hline & & & & $\mathrm{O}^{*}$ & $\mathrm{G}^{*}$ & $\mathbf{B}^{*}$ & $\mathrm{D}^{*}$ & \\
\hline 1 & E148Q /- & & & & + & + & & 5 \\
\hline 2 & E148Q /- & & & & + & & + & 8 \\
\hline 3 & E148Q /- & & & & + & & & 6 \\
\hline 4 & E148Q / - & & & + & + & & & 5 \\
\hline 5 & E148Q /- & & & & + & + & + & 6 \\
\hline 6 & E148Q / - & & & Sin endoscopía & 7 & & & \\
\hline 7 & E148Q / - & & & & + & & & 6 \\
\hline 8 & E148Q / - & & & + & + & & & 7 \\
\hline 9 & E148Q / - & & & & + & + & + & 8 \\
\hline 10 & E148Q / - & & & & + & & & 6 \\
\hline 11 & & & V726A / - & + & + & & & 6 \\
\hline 12 & & & V726A / - & & + & & & 6 \\
\hline 13 & & & V726A / - & + & & + & & 6 \\
\hline 14 & & & V726A / - & Sin endoscopía & 5 & & & \\
\hline 15 & R202Q / - & & V726A / - & & + & & & 7 \\
\hline 16 & R202Q / - & & & & + & & & 9 \\
\hline 17 & R202Q / - & & & + & + & + & + & 8 \\
\hline 18 & R202Q / - & & & + & + & & & 6 \\
\hline 19 & R202Q / - & & & & + & & + & 7 \\
\hline 20 & & & M694V /- & & + & & + & 8 \\
\hline 21 & & & M694V /- & & + & & & 5 \\
\hline 22 & & & M694V /- 1 & Hallazgos endoscópicos normales & 6 & & & \\
\hline 23 & & P369S /- & & Sin endoscopía & 5 & & & \\
\hline 24 & & & K695R / - 1 & Hallazgos endoscópicos normales & 6 & & & \\
\hline 25 & & & $\mathrm{R} 761 \mathrm{H} /-$ & Sin endoscopía & 5 & & & \\
\hline 26 & & & $\mathrm{R} 761 \mathrm{H} /-$ & & + & & & 6 \\
\hline 27 & & $\mathrm{R} 314 \mathrm{R} /-$ & & & + & + & & 7 \\
\hline 28 & & R314R/- & & + & & & & 6 \\
\hline 29 & & & P706P / - & + & & & & 5 \\
\hline 30 & & & P124P / - & + & + & & & 7 \\
\hline 31 & R202Q / R202Q & & & Sin endoscopía & 6 & & & \\
\hline 32 & R202Q / R202Q & & & Hallazgos endoscópicos normales & 6 & & & \\
\hline 33 & R202Q / R202Q & & & & + & & & 5 \\
\hline 34 & R202Q / R202Q & & & & & + & + & 6 \\
\hline 35 & R202Q / - & & M694V/- & + & + & + & + & 8 \\
\hline 36 & R202Q / - & & M694V/- & + & & & & 5 \\
\hline 37 & & & M694V/M694V & + & + & + & + & 12 \\
\hline 38 & & & M694V/M694V & + & & + & + & 8 \\
\hline 39 & R202Q / - & & M680I /- & & + & & & 10 \\
\hline 40 & E148Q / - & & M680I /- & & + & & & 5 \\
\hline 41 & R202Q / E148Q & & & Sin endoscopía & 7 & & & \\
\hline 42 & L110P / E148Q & & & & & & + & 7 \\
\hline 43 & E148Q/E148Q & & M694V /- & Sin endoscopía & 6 & & & \\
\hline 44 & E148Q/E148Q & & M694V /- & + & + & + & + & 11 \\
\hline 45 & E148Q/E148Q & & M694V /- & + & + & + & + & 12 \\
\hline 46 & R202Q/R202Q & & M694V /- & + & + & + & + & 12 \\
\hline 47 & Mutación negativa, FI & & & & + & & + & 8 \\
\hline 48 & Mutación negativa, FI & & & + & + & & & 6 \\
\hline
\end{tabular}

${ }^{\star}$ E: esofagitis, G: gastritis, B: bulbitis, D: duodenitis.

${ }^{* *}$ DSS: puntaje de severidad de la enfermedad. 
realizado por Gurkan y col. ${ }^{12}$ En su estudio, durante la evaluación endoscópica, se observó inflamación colónica y gástrica. Se ha explicado el compromiso digestivo debido a la proliferación bacteriana relacionada con la dismotilidad y la absorción insuficiente causadas por el depósito de amiloide. Sin embargo, en ese estudio, no se estableció una relación entre la inflamación de la mucosa y la amiloidosis.

La colchicina mejora los síntomas de la enfermedad, pero en el caso de síntomas digestivos relacionados con la FMF, se debe considerar la resistencia a este fármaco. ${ }^{13}$ Es posible determinar la presencia de características histológicas gástricas típicas, como seudoproliferación epitelial, agotamiento de mucina y apoptosis, en los pacientes con FMF. ${ }^{14}$ En nuestro estudio, la endoscopía digestiva alta reveló esofagitis, gastritis, bulbitis y duodenitis en todos los pacientes, excepto por tres pacientes que tuvieron resultados normales. En la evaluación histológica de las biopsias, no se observaron cambios secundarios a la colchicina. En seis pacientes con DSS alto según Pras, ${ }^{7}$ se observó inflamación moderada o grave desde el esófago hasta el duodeno. Por otro lado, los pacientes con mutaciones homocigotas tenían DSS más altos. No obstante, debido a la falta de un grupo de referencia y a la cantidad pequeña de pacientes, esta diferencia no se consideró estadísticamente significativa y no pudo interpretarse. En la

FIGURA 2. Hallazgos endoscópicos de esofagitis (paciente núm. 35)

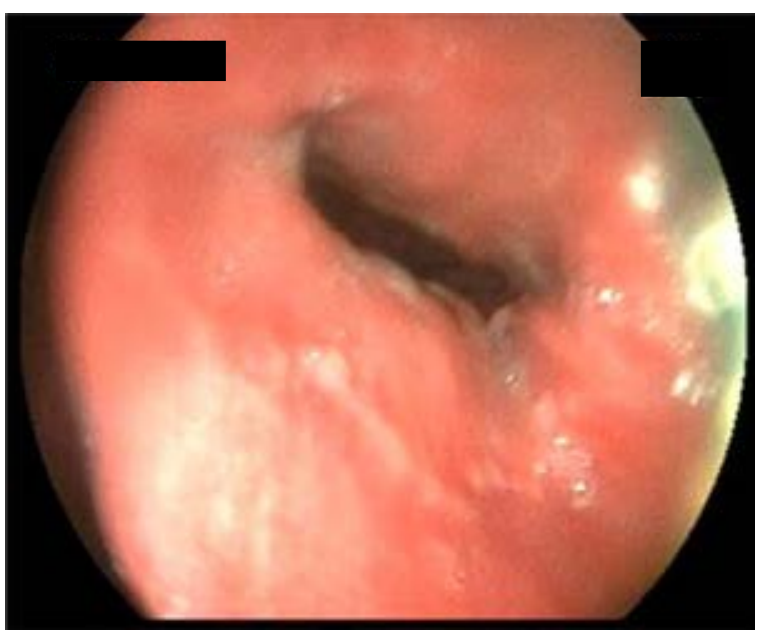

Erosiones que afectan la región desde la porción distal del esófago hasta la línea $Z$ de la unión gastroesofágica con un patrón rayado. bibliografía, se describe un estudio en adultos en el que se evaluaron los síntomas digestivos de la FMF mediante cápsula endoscópica. De manera similar a nuestro estudio, se observaron defectos pequeños en la mucosa intestinal, como erosiones y edema. ${ }^{15}$

En este estudio, se determinó por histología la presencia de infección por H. pylori en todos los pacientes con resultados positivos en la prueba del aliento con urea marcada. No se ha verificado que los hallazgos clínicos e histológicos en un paciente con serología positiva indiquen la presencia de celiaquía. En el $67 \%$ de los pacientes con inflamación digestiva se observó una respuesta positiva al tratamiento. Entre las limitaciones más importantes de este estudio, se deben mencionar la cantidad relativamente pequeña de pacientes y la falta de registro de los efectos secundarios de la colchicina.

Como resultado, a los pacientes que recibieron una dosis efectiva de colchicina y que tuvieron marcadores de inflamación normales en los análisis de laboratorio y con dolor abdominal con características clínicas diferentes a las de los ataques típicos de FMF, se los debe someter a una evaluación gastroenterológica. Las intervenciones endoscópicas cumplieron una función informativa en este grupo de pacientes. Debe verificarse la correlación positiva probable entre la inflamación digestiva histológicamente grave y el DSS más alto en una serie más amplia.
FIGURA 3. Alteraciones macroscópicas que varían de eritema a erosiones en la mucosa del duodeno.

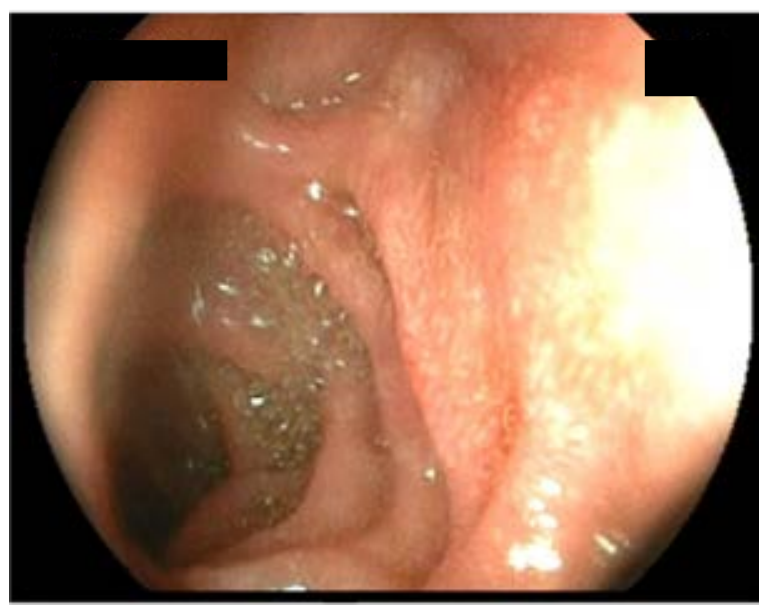




\author{
Agradecimientos \\ Quisiéramos agradecer a Guzide Aksu por su \\ apoyo y ánimo.
}

\section{REFERENCIAS}

1. Brydges S, Althreyra B, Kastner DL. Periodic fever syndromes in children. En: Cassidy JT, Petty RE, Laxer RM, et al eds. Textbook of pediatric rheumatology. 5th ed. Philadelphia: Elsevier-Saunders; 2005.Págs.657-67.

2. Livneh A, Langevitz $P$, Zemer $D$, et al. Criteria for the diagnosis of familial Mediterranean fever. Arthritis Rheum 1997; 40(10):1879-85.

3. Ben-Chetrit E. Familial Mediterranean fever (FMF) and renal AA amyloidosis-phenotype-genotype correlation, treatment and prognosis. J Nephrol 2003; 16(3):431-4.

4. Mor A, Gal R, Livneh A. Abdominal and digestive system associations of familial Mediterranean fever. Am J Gastroenterol 2003; 98(12):2594-604.

5. Mor A, Shinar Y, Zaks N, et al. Evaluation of disease severity in familial Mediterranean fever. Semin Arthritis Rheum 2005; 35(1):57-64

6. Sari S, Egritas O, Dalgic B. The familial Mediterranean fever (MEFV) gene may be a modifier factor of inflammatory bowel disease in infancy. Eur J Pediatr 2008; 167(4):391-3.

7. Pras E, Livneh A, Balow JE Jr, et al. Clinical differences between North African and Iraqi Jews with familial Mediterranean fever. Am J Med Genet 1998; 75(2):216-9.
8. Baber KF, Anderson J, Puzanovova M, et al. Rome II versus Rome III classification of functional gastrointestinal disorders in pediatric chronic abdominal pain. J Pediatr Gastroenterol Nutr 2008; 47(3):299-302.

9. Abuhammour A, Dajani A, Nounou M, et al. Standard triple therapy versus sequential therapy for eradication of Helicobacter pylori in treatment naïve and retreat patients. Arab J Gastroenterol 2016; 17(3):131-6.

10. Ozturk C, Halicioglu O, Coker I, et al. Association of clinical and genetical features in FMF with focus on MEFV strip assay sensitivity in 452 children from western Anatolia, Turkey. Clin Rheumatol 2012; 31(3):493-501.

11. Kiss MH, Magalhães CS. Autoinflammatory diseases: mimics of autoimmunity or part of its spectrum? Case presentation. J Clin Immunol 2008; 28 Suppl 1:S84-9.

12. Gurkan OE, Dalgic B. Gastrointestinal mucosal involvement without amyloidosisin children with familial Mediterranean fever. J Pediatr Gastroenterol Nutr 2013; 57(3):319-23.

13. Kallinich T, Haffner D, Niehues T, et. al. Colchicine use in children and adolescents with familial Mediterranean fever: literature review and consensus statement. Pediatrics 2007; 119(2):e474-83.

14. Al-Daraji WI, Al-Mahmoud RM, Ilyas M. Gastric changes following colchicine therapy in patients with FMF. Dig Dis Sci 2008; 53(8):2079-82.

15. Demir A, Akyüz F, Göktürk S, et al. Small bowel mucosal damage in familial Mediterranean fever: results of capsule endoscopy screening. Scand J Gastroenterol 2014; 49(12):1414-8. 